\title{
Time to Compile
}

\section{Catie Cuan \\ Independent Artist \\ Brooklyn, NY \\ crcuan@gmail.com}

\author{
Amy LaViers \\ Department of Mechanical Science and \\ Engineering, University of Illinois at Urbana- \\ Champaign \\ alaviers@illinois.edu
}

\section{Ishaan Pakrasi}

Department of Mechanical Science and

Engineering, University of Illinois at Urbana-

Champaign

pakrasi2@illinois.edu

\section{ABSTRACT}

"Time to Compile" ${ }^{1}$ is the result of an extended in-house residency of an artist in a robotics lab. The piece explores the temporal and spatial dislocations enabled by digital technology and the internet and plays with human responses to articulated machines (robots) in that setting. The audience journeys through a suspended, disparate landscape that aims to reconcile these responses to technology and machines. This proposal offers to bring an excerpt of the piece, live dance performance surrounded by videos of robots created in the lab, to MOCO. Additionally, an interactive installation could be produced if MOCO has the timing bandwidth to offer this more involved setup.

Permission to make digital or hard copies of part or all of this work for personal or classroom use is granted without fee provided that copies are not made or distributed for profit or commercial advantage and that copies bear this notice and the full citation on the first page. Copyrights for third-party components of this work must be honored. For all other uses, contact the owner/author(s).

MOCO, June 28-30, 2018, Genoa, Italy

(C) 2018 Copyright is held by the owner/author(s).

ACM ISBN 978-1-4503-6504-8/18/06

https://doi.org/10.1145/3212721.3212888 


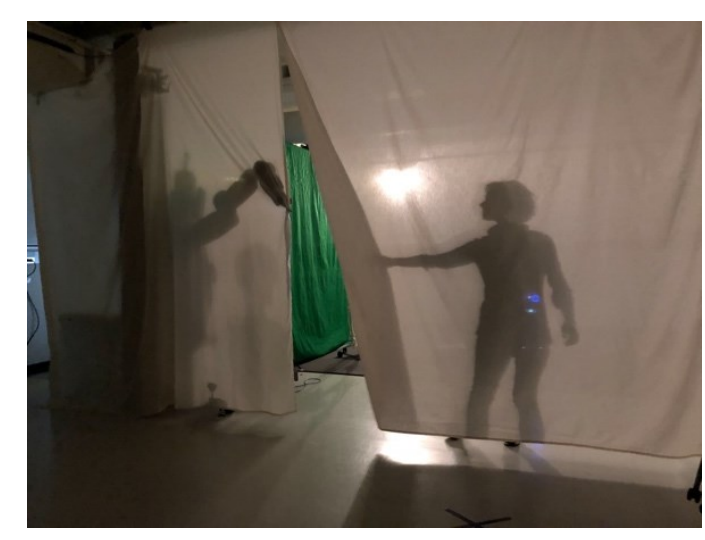

Figure 1: Stage elements used

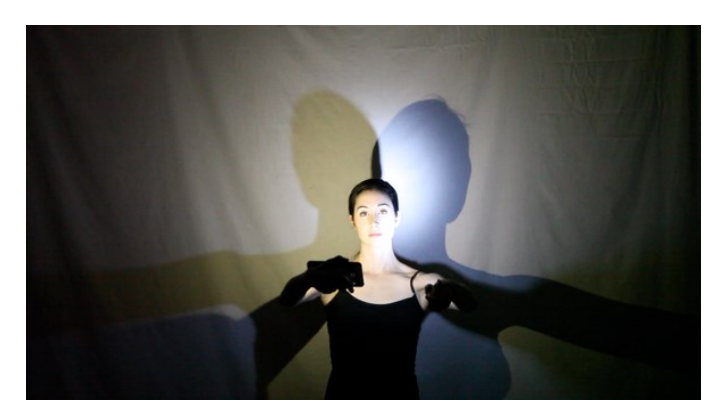

Figure 2: Stage elements used

\section{CCS CONCEPTS}

- Applied computing performing arts;

\section{KEYWORDS}

dance, video, performance, internet, connection, robotics, trust, valence, human-robot interaction

ACM Reference format:

C. Cuan, I. Pakrasi and A. LaViers. 2018. Time to Compile. In MOCO, June 28-30, 2018, Genoa, Italy, 4 pages. DOI: $10.1145 / 3212721.3212888$

\section{INTRODUCTION}

The internet is no longer the separate, computational space it has been the past 4 decades; more devices are hooked to the internet than people (and $50 \%$ percent of the global population is connected). In building this massive network, engineers have focused on how individual widgets and mechanisms, can be interconnected to create addictive functional devices; what has been underexamined is sincerity, or, in other words, the human relationship to these resultant tools [2]. With our data constantly being recorded on various platforms, digital manifestations of our identities exists in servers across the world unbeknownst to us. These fractured, quantitative digital identities often create messy, confusing translations in "real life" as explored in [1]. Choreographers have much to contribute to this conversation as in [3]. "Time to Compile" is an embodied analog for the Internet of Things where performers (including dancers and engineers) help audience members make sense of this "place" and this "time". The mood of the piece is distant and remote yet sparkly and intriguing. We use soft elements (both live and pre-recorded) like sheets, skin, and sex to contrast the hard lines of robots, virtual avatars, and other digitally controlled devices. Technologies like film, dance, and live streaming are used to usher the audience into this suspended, provocative, and everchanging environment.

\section{ARTISTIC THEMES}

"Time to Compile" was thus created in response to three topics of concern: 1) The Hidden Human Network. Many technologies are powered by humans for the benefit of each other, but often this network is occluded, leaving a machine seeming quite intelligent, e.g., IBMs Watson, which is powered by the webpage postings of users all over the Internet. 2) Are humans becoming more robotlike? This question was originally posed in the reverse, but upon further inspection, it is easy to argue that the rich adaptability of humans is heavily exploited in emerging technologies (more than any particularly successful imitation of biology). 


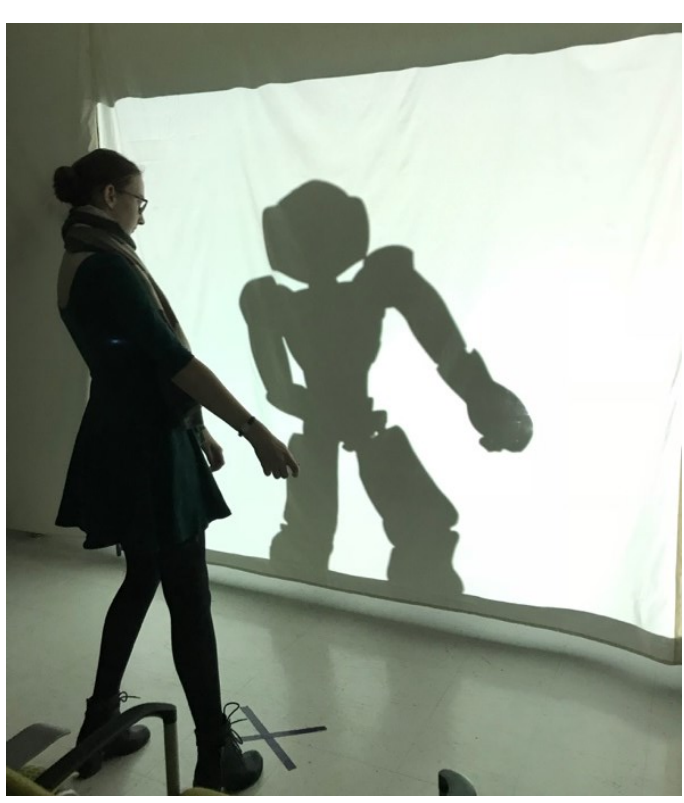

Figure 3: Embodied exercise
With these changes, are humans finding social structures like family or friendships in embodied and personal technology experiences? 3) Time to Compile. How long does it take to find resolution with or understanding of different technologies? How long before we iterate on the first design and find a second? Who gets to investigate the inner-workings of these machines before? When have we assimilated a new technology permanently? How will we change? The aim of "Time to Compile" is to reveal the hidden human network and to alter the objective action of the participants, testing the hypothesis that human plasticity can be exploited through technology. As the hidden human network is unmasked, participants recognize how this embodied illustration of the network can be reflected across the spectrum of their daily life. For example, self-driving cars are in fact machines created by humans and programmed by humans, with biases from the teams that made them. It follows simply from these machines are limited to their programmed capabilities.

\section{HUMAN-ROBOT INTERACTION USER STUDY}

To understand how viewing "Time to Compile" can alter an audience's views on robotics, choreography, and the future. We created an experimental testbed format to explore this question. An initial survey was created for the audience to complete in advance of viewing "Time to Compile". This survey explores questions related to the power, valence, and acceptability of various agents that will appear in "Time to Compile". A secondary survey was created for the audience to complete after a viewing of "Time to Compile", with many similar questions and a few additions. In instances where there is a multi-part instantiation of "Time to Compile", additional surveys are added in the intervening section changes.

\section{AUDIENCE EXPERIENCE}

"Time to Compile" is generally comprised of a staged performance and embodied, interactive exercises. Figures 1 and 2 provide examples of stage performed elements. Figures 3 and 4 provide examples of embodied, installation-based exercises. Both the staged performance and embodied exercises may include the research surveys as outlined in the section above. Therefore either or both can fit within the user study testbed format. The staged performance involves several pre-recorded and live elements including videos, dialogue, and dance. The first section has a hypnotic and magical quality, priming the audience with deliberate, complicated interactions with robotic technology. There is an exploration with shadow play, chaotic music, and extreme close ups on video. The embodied interactive installation invites audience participants to follow various "agents", sometimes including a shadow video, a human, and a VR avatar. 


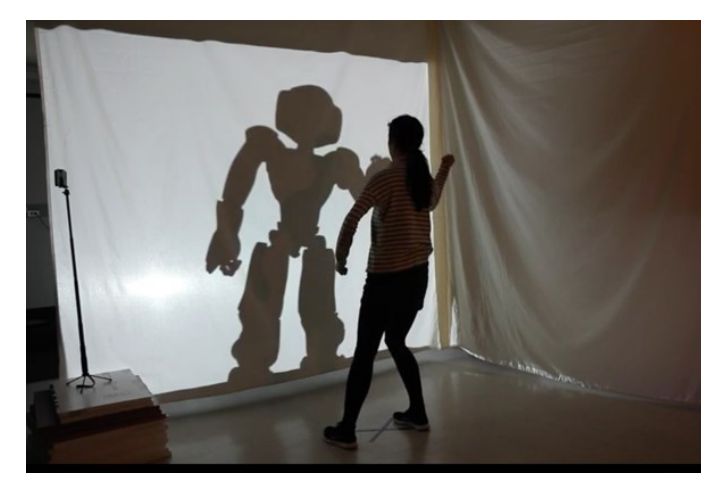

Figure 4: Embodied exercise

These experiences symbolize the various embodied interactions that happen in the digital and real world throughout our daily lives. The interactions oscillate between simple and frustrating, simulating the feeling of alienation and satisfaction we often experience with machine and computer interfaces. The overall tone is safe, exploratory, and suspended. Audience members often express that these exercises feel like a journey.

\section{REFERENCES}

[1] Ken Goldberg. 2001. The Robot in the Garden: Telerobotics and Telepistemology in the Age of the Internet. MIT Press.

[2] Dan Nosowitz. 2014. Nest Recalls Nearly Half A Million Of Their Protect Smoke Detectors Due To Design Flaw. Fast Code Design (May 2014). https://www.fastcodesign.com/3030954/

[3] Thecla Schiphorst. 2013. Merce Cunningham: Making dances with the computer. Merce Cunningham: Creative elements (2013), 79-98 